\title{
Acad. Prof. Em. Eq. Mayor (M) Alfredo Ubaldo Ramón-Guerra Carámbula Primer Jefe del Servicio de Pediatría del Hospital Militar (1962-1969)
}

\author{
doi http://dx.doi.org/10.35954/SM2020.39.1.1 \\ Dr. Augusto Soiza Larrosa (D) https://orcid.org/0000-0002-3924-9976 \\ Médico. Miembro del Instituto Histórico y Geográfico del Uruguay. \\ Miembro de Honor y ex presidente de la Sociedad Uruguaya de Historia de la Medicina.
}

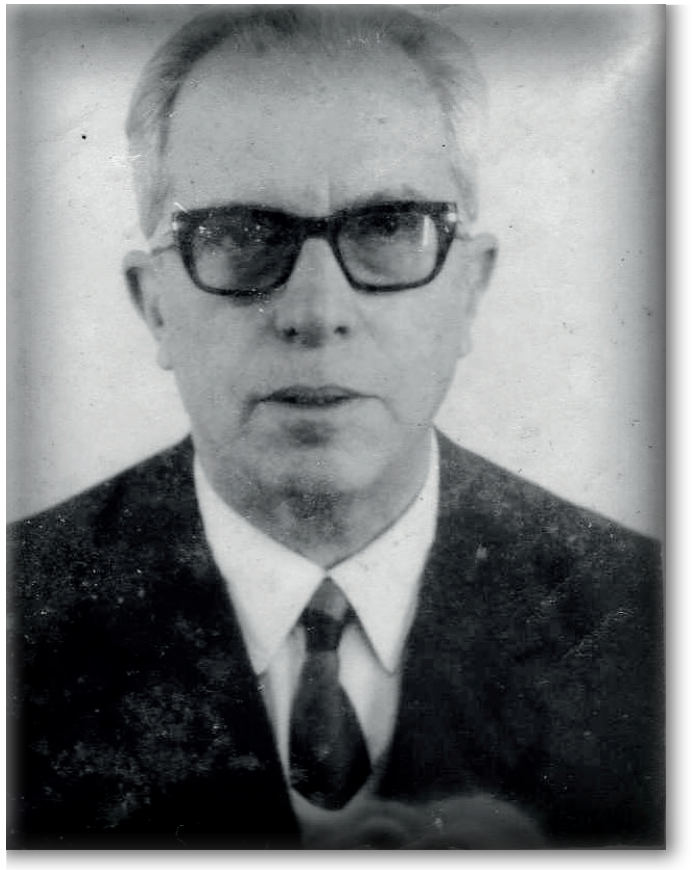

Figura 1. Primer Jefe del Servicio de Pediatría del Hospital Militar 1962-1969.

Alfredo Ubaldo Ramón-Guerra Carámbula nació el 20 de julio de 1904, en la ciudad de Las Piedras $4^{a}$ sección del Departamento de Canelones, en el domicilio de sus padres Don Ubaldo Ramón-Guerra y Doña Bernabela Carámbula ambos orientales; según consta en el certificado del Registro de Estado Civil que he tenido a la vista. Fueron sus abuelos paternos, José María Ramón y Basilia Guerra, también orientales; y por el lado materno, Benigno G. Carámbula de profesión militar, y Petrona Acosta, asimismo orientales. Su padre, fue diplomático y representó al Uruguay ante el Gobierno de Italia en los años previos a la Segunda
Guerra Mundial. Alfredo Ramón-Guerra casó en Montevideo en octubre de 1947 con Margarita Minikes, nacida en Basel [Basilea], Suiza, frontera con Alemania. Sus dos hijos fueron María Cristina (1948) y Sylvia (1950). Falleció en Montevideo el 10 de enero de 1996. Fue una figura brillante de la pediatría uruguaya, de muy sólida formación clínica y básica. Pero también fue un maestro. Un creador infatigable por el bienestar del niño, que tuvo grandes logros. No para beneficio personal, ni para acceder a cargos de cualquier naturaleza, universitarios o gubernamentales. Los beneficios de sus creaciones perviven hasta hoy, aunque la mayoría de los ciudadanos y especialmente de los médicos en ejercicio, los desconozcan. Por eso viene bien refrescar la rica historia de un médico uruguayo ejemplar, en su dignidad, sabiduría y valores éticos. Hizo su formación de posgrado en Europa, en afamados centros de Alemania, Francia e Italia, así como en los Estados Unidos de América. Se interesó en profundidad no sólo por los problemas vinculados a la medicina y la pediatría, sino también de las ciencias básicas, particularmente la físico-química. Hombre de vasta cultura general fue un exquisito cultor de la música clásica. Su producción científica abarcó más de 355 publicaciones. Fue miembro fundador de la Academia Nacional de Medicina, de la genética clínica en el Uruguay; primer director del Departamento Materno-Infantil del Ministerio de Salud Pública (MSP); creador del "Plan Aduana" de referencia y contra-referencia en la atención del 
niño, base de la atención primaria de salud en la comunidad. Iniciador de los cuidados intensivos pediátricos en el Uruguay. Profesor de Patología Médica de la Facultad de Medicina. Una de las figuras de más amplio magisterio y de mayor brillo intelectual de la pediatría en el Uruguay del siglo $X X$. De amplio reconocimiento regional e internacional, con membresías y honores en la casi totalidad de las Sociedades de Pediatría de América Latina y Miembro de la Academia Americana de Pediatría (1).

\section{Su inicial desempeño en el Servicio de Sanidad Militar}

Ramón-Guerra recibió su título de médico cirujano de la Facultad de Medicina de Montevideo en 1930. Estuvo en Europa hasta 1936 y a su retorno, con un preciado bagaje de conocimiento científico, comenzó su prestigiosa carrera pediátrica. Tempranamente se vinculó a la entonces llamada Sanidad Militar (S.S.FF.AA.), que era, con la Facultad de Medicina y el Ministerio de Salud Pública, uno de los cargos médicos apetecidos. La historia de la asistencia pediátrica en el Hospital Militar (H.C.FF.AA.) no ha sido escrita. En 1924 existía una policlínica de niños a cargo del doctor Antonio Bargo, y desde 1927 del profesor agregado de la Clínica de Niños de la Facultad de Medicina, Víctor Zerbino (1888-1943). Los cuadros quirúrgicos se asistían en el Servicio de cirugía de mujeres y niños.

1940: "Visto el oficio de la Dirección General de Sanidad Militar, donde propone la designación de Médicos Honorarios con el propósito de mejorar los servicios de policlínicas de Vías Urinarias y de Niños del Hospital Militar Central, el Presidente de la Repúblicas decreta, nómbrase...".

Se designaron respectivamente a los doctores Emilio Bonnecarrére y Alfredo U. Ramón-Guerra (decreto No 806 del 25/9/1940).

En 1941 ingresó al escalafón civil del Servicio de Sanidad Militar y firmó el siguiente oficio:
"Montevideo, 6 de agosto de 1941.

Señor Director General de la Sanidad Militar General de División Retirado Don José M. López Vidaur Señor Director:

Tengo el honor de acusar recibo de su nota No 1966, de fecha 27 de Julio último, por la que me hace saber que a propuesta de esa Dirección General, el Poder ejecutivo, por Decreto No 1131 del día 10 de Julio ppdo. Me designó Oficial 9o (Médico de Batallón) Categoría III, Grado 7, del Item No 3.19 - Sanidad Militar.

Al agradecer al señor Director General el nombramiento de la referencia, aprovecho esta oportunidad para saludarlo con mi consideración más distinguida

\section{(rúbrica) Alfredo U. Ramón Guerra" (2).}

El alta presupuestal es del 07 de setiembre de 1941 con asignación mensual de 110 pesos. En noviembre de ese año el Director General le calificó con el siguiente juicio "en su corta duración ha demostrado gran competencia y laboriosidad". Tenía entonces 37 años, y desde su regreso de Europa en 1936 se había vinculado al Servicio de Pediatría del profesor Salvador Burghi (1874-1950) en el hospital pediátrico "Pedro Visca", frente al Asilo Dámaso Antonio Larrañaga. Ramón-Guerra no sólo fue su discípulo y colaborador desde 1936 (Médico Ayudante por concurso de oposición entre 1939-41) sino su amigo, con el cual disfrutaba los almuerzos caseros de los jueves en la casa de Burghi con otros colegas (Conrado Pelfort, Euclides Peluffo, Héctor Bazzano, Ruben Gorlero Bacigalupi) y las pescas en chalana en otoño y primavera en Punta del Este (3).

En setiembre de 1942 solicitó licencia para concurrir como miembro informante uruguayo al XII Congreso de Pediatría en Buenos Aires.

En 1945 ascendido a Oficial 5 Médico y en 1946 aparece su opción por tomar estado militar según el estatuto aprobado por el Poder Legislativo respecto a los Servicios Auxiliares de las Fuerzas Armadas. El 22 de enero de 1948 renunció al cargo de médico pediatra del Hospital Militar (2). 


\section{Postulación como Jefe de Servicio de Pediatría}

\section{"Montevideo, Julio 26 de 1962.}

Señor Jefe de la Segunda División Organización del Servicio de Sanidad Militar

Alfredo Ramón Guerra, Médico Pediatra, Credencial Cívica A.I.A. No. 3171, domiciliado en Rambla República del Perú No. 1163 Apto. 10, solicita ser inscripto en el concurso para llenar el cargo de Jefe de Servicio de Pediatría del Hospital Militar Central, acompañando al presente por duplicado los méritos, trabajos y servicios prestados.

La documentación que establece el artículo 6to. Del Decreto No. 23.858 obra en su legajo personal.

Saluda a Ud. atentamente

Médico Pediatra

\section{(rúbrica) Dr. Alfredo Ramón Guerra"}

Se exigía una constancia de dos profesionales acerca del conocimiento de sus virtudes personales, y la misma fue extendida por el doctor Rodolfo Saccone Erosa (1920-2012), cirujano del Hospital Militar entre 1955 y 1987 y Carlos Alfredo Escande García (1919-1973), médico pediatra del Hospital Militar entre 1961 y 1973, falleciendo en el desempeño de su cargo de Jefe del Servicio de Pediatría:

"Los que suscriben Dres. Carlos A. ESCANDE y Rodolfo SACCONE, credencial cívica serie BLB No.7538 y credencial cívica serie AXA No. 6238, vecinos de este Departamento, domiciliados en Montevideo, Certifican: Que desde el año 1945 conocen al Dr. Alfredo RAMÓN GUERRA y pueden dar fe que desde 1945 hasta el presente, ha observado conducta intachable y de que profesa los ideales democráticos y republicanos. A pedido del interesado y al efecto de su ingreso al Servicio de Sanidad Militar expedimos el presente en Montevideo, a los veintisiete días del mes de julio del año mil novecientos sesenta $y$ dos.

(rúbrica) Dr. Carlos A. Escande

(rúbrica) Dr. Rodolfo Saccone

Domiciliados:

Larrañaga 2776 -ap. 2

Rbla. O'Higgins No. 5171".
Fue nombrado Jefe del Servicio de Pediatría por Resolución Ministerial № 32.169 del 02 de octubre de 1962 (Boletín del Ministerio de Defensa Nacional (MDN) N 5206) en el ítem 3.19 "Servicio de Sanidad Militar", Categoría I, Grado 5 (Orden de la Dirección General No 420 del 11/10/1962) con una asignación mensual de 2.550 pesos. Tomó posesión del cargo el 11 de octubre de 1962, según lo comunicó por Oficio el Director General del Servicio de Sanidad Militar coronel José C. López al Jefe de la División Personal. Ese mismo año estuvo de visita en Montevideo, el profesor agregado de la Facultad de Medicina de París Pierre Royer, pediatra y director de la Unidad de Investigación del INSERM (Instituto Nacional Francés de Salud e Investigación Médica) en enfermedades del metabolismo en el niño en el Hôpital des Enfants Malades de Paris. Mantuvo una cordial relación con el doctor Ramón-Guerra, dejando constancia de su agradecimiento por acompañarlo e invitándolo a su servicio en 1963. La carta invitación de Pierre Royer llevó fecha 25 de noviembre de 1962:

"Docteur Pierre ROYEUR

Professeur Agrége A La Faculté de Médecine

9, Rue de Villersexel

Littre 31-32

Le 25 de noviembre

Cher docteur Ramón Guerra.

Je vou lois vous remercier de l'extreme gentillesse de votre accueil a Montevideo. J'aiètè trés impresionè pour la qualitè du travail qui est rèalisè..."

Siendo Ramón-Guerra miembro de la Comisión de Reestructura de los Servicios Generales del Hospital Militar solicitó licencia para trasladarse a París, atendiendo a la invitación del profesor Royer y se le encomendó un informe sobre la asistencia pediátrica en los hospitales militares que visitara durante su estadía europea.

Del Servicio del profesor Royer nos ha dejado un recuerdo el profesor José Grunberg (figura 2), que fuera Jefe del Departamento de Pediatría del H.C.FF.AA. sucediendo al profesor Carlos A. Escande: 


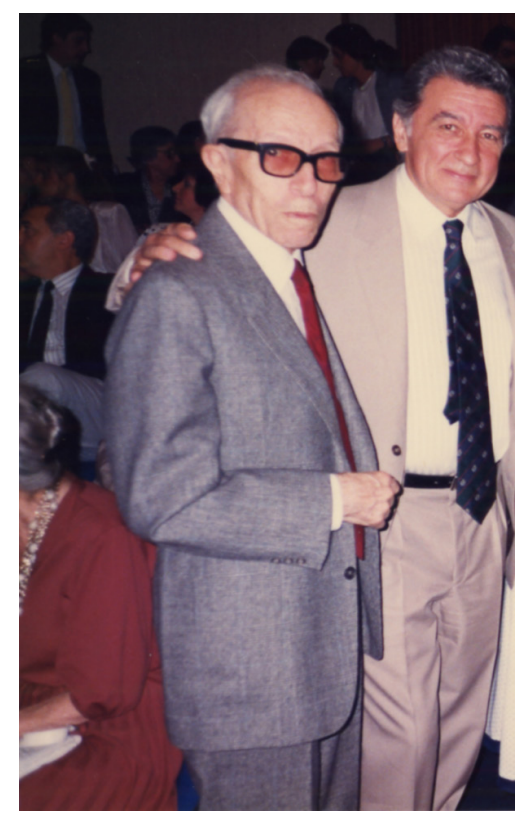

Figura 2. Prof. Alfredo U. Ramón-Guerra con el Dr. José Grunberg

"El Maestro y amigo Ramón Guerra, una persona decisiva en mi vida científica, me relacionó con el Prof. Pierre Royer, del Hôpital des Enfants Malades. Llegamos con un hijo de cinco años, a un gélido Paris en enero de 1963. En el hospital puse en acción "mi" francés; mera ilusión. Los colegas destinatarios de mi dialéctica, dudaban cual era el idioma que hablaba. Comprobé, estupefacto, que el famoso servicio de Pierre Royer era pobre en recursos materiales, con ostensible precariedad edilicia. El equipamiento tecnológico, era muy inferior al que disponíamos en Montevideo. Un ejemplo; carecían de laboratorio con micro métodos. ¡En Montevideo estudiábamos el equilibrio ácido base en una gota de sangre, en Paris requerían $10 \mathrm{ml}$ ! Reconocí la pujanza y visión de Ramón Guerra en la introducción de la tecnología de punta a nuestro medio, con un colaborador, Sarandí Bidegain, que llevaba ideas y tecnología a la práctica corriente, inolvidable por su generosidad y entrega. El microscopio de la patóloga, la Dra. Renée Habib, que revolucionó la taxonomía internacional de la anatomía patología renal moderna, no era diferente del nuestro. Más allá de lo que aprendí de la nefrología pediátrica en Servicio de Pierre Royer, fui testigo y alumno del desarro- llo de un modelo de generación de conocimiento, de la motivación por la investigación y la innovación, del valor del "full time" geográfico y de la dinámica y organización del trabajo en equipo" (4).

En 1964 solicitó licencia para trasladarse a las ciudades de Valencia y Caracas (República de Venezuela) para dictar conferencias en la Cátedra de Puericultura y Pediatría de la Universidad de Carabobo y en el Hospital del Niño de Panamá sobre genética, nutrición y patología del recién nacido. En 1967 hizo las gestiones para trasladarse a la ciudad de Barranquilla (Colombia) por invitación oficial al Congreso de Pediatría.

\section{Retirado militar}

En 1969, siendo Jefe del Servicio de Pediatría como funcionario civil solicitó ser comprendido en el escalafón militar de Servicios Auxiliares (Bf) de acuerdo a las leyes 13.737 (art. 38) y 13.640 (art. 53). De acuerdo a las mismas, se le reconocían los servicios prestados desde su reingreso como Jefe de Servicio con estado militar. Por su edad y antigüedad (8 años, 7 meses, 22 días desde el nombramiento en 1962) le correspondía el grado de teniente $1^{\circ}$, grado que le fue concedido el 01/07/1969 (Boletín MDN Nº 6.156), pero también el pase a retiro por edad (mayor de 64 años). Así, con fecha retroactiva al 07/05/1968 fue incorporado al escalafón militar "en situación de retiro" conjuntamente con el entonces Jefe del Servicio de Urología doctor Frank Antonio Hughes Lussich. Le sucedió como Jefe del Servicio de Pediatría el doctor Carlos Alfredo Escande García (19191973) quien accedió al cargo por concurso de méritos con fecha 04/02/1970 (5).

\section{Centro de Tratamiento Intensivo Pediátrico del Hospital Militar}

En 1972 fue propuesto como asesor del proyectado Centro de Tratamiento Intensivo Pediátrico del Hospital Militar. Y lo fue en virtud de "desempeñarse en el Centro de Tratamiento Intensivo del Hospital Pereira Rossell y estando abocada 
la Sanidad Militar a inaugurar un centro similar, donde su reincorporación reportará beneficios como asesor, coordinador y normatizador" (oficio del Director Técnico del Hospital al Director del Servicio de Sanidad de las Fuerzas Armadas (S.S.FF.AA.), 29/12/1972). En consecuencia fue designado por Resolución Ministerial N $N^{\circ} 46.250$ del 23/01/1973 (Boletín MDN № 6.624) en el S.S.FF.AA. como teniente $1^{\circ}$ en situación de retiro y equiparado al grado de mayor por Resolución Ministerial № 47.209 del 16/10/1973 (Boletín MDN N 6.722) pero "debiendo permanecer en situación de retiro". El 18 de junio de 1973 en el marco de creación de los Departamentos Médicos se creó el Departamento de Pediatría designándose como jefe al doctor profesor Carlos A. Escande y como adjunto a la dirección al Mayor (SM-M) Rodolfo Maggi. Lamentablemente, el doctor Escande, ya enfermo, falleció el 12 de agosto de 1973.

\section{Retiro definitivo del doctor Alfredo Ramón-Guerra}

El 11 de junio de 1983 pasó a situación de retiro habiendo computado 37 años de servicio por Resolución Ministerial № 61.357 del 28/9/1983 (Boletín MDN No 2.251). Por considerarlo de interés para su biografía, inserto la cronología de los servicios prestados por el doctor Alfredo U. Ramón-Guerra en el MSP, según copia del informe $N^{\circ} 475$ de dicho ministerio del 27/1/1969 expedido a pedido del interesado, y que se encuentra a fs. 128 de su legajo de la Dirección Nacional de Sanidad de las Fuerzas Armadas (2):

1937: Médico Asistente (autorizado) en el Servicio de Lactantes "C" del Hospital Pedro Visca

17/06/1937 a 22/03/1939: Médico Ayudante del Servicio de Lactantes (int) del Hospital Pedro Visca 23/03/1939 a 06/08/1941: Médico Ayudante del Servicio de Lactantes (por concurso de oposición) del Hospital Pedro Visca

1942 a 1948: Médico Asistente (autorizado) en el Servicio de Lactantes "C" del Hospital Pedro Visca
21/03/1947: Encargado del Servicio de Lactantes "C" del Hospital Pedro Visca

17/02/1954 a 13/4/1955: Médico Jefe del Servicio de Lactantes (I) del Hospital Pedro Visca

06/08/1954: concurre al Congreso de Pediatría de San Pablo, República Federativa de Brasil

21/09/1955 a 28/05/1957: Médico Jefe de Sala y Policlínica Pediátrica (int), del Hospital Pedro Visca 06/11/1956: concurre al Seminario de Protección a la Infancia (Chile y Perú)

04/06/1957 a 10/01/1958: Médico de Endocrinología (int) del Hospital Pereira Rossell

16/01/1958 a 12/02/1959: Director del Departamento de Nutrición, Alimentación y Vitaminología (int) de la División Higiene del MSP

19/10/1962: presidente de los tribunales de concurso para Médico de Guardia de los Hospitales Pedro Visca y Pereira Rossell

24/10/1967: coordinador de la Unidad de Tratamiento Intensivo Pediátrico del Hospital Pereira Rossell 18/12/1967: integrante de la Comisión Asesora del Plan Nacional de Lucha contra la Diarrea Infantil

\section{REFERENCIAS}

(1) Turnes A. Alfredo U. Ramón Guerra (1904-1996). Disponible en: https://www.smu.org.uy/dpmc/hmed/ historia/articulos/ramon-guerra.pdf [Consulta 19/12/2019].

(2) Uruguay. Dirección Nacional de Sanidad de las Fuerzas Armadas. Dpto. de Personal. Archivo. Legajo № 6735 , carpeta № 165 . Alfredo U. Ramón Guerra.

(3) Brazeiro Diez H. Salvador Burghi (1874-1950). 5 páginas. Disponible en: https://www.smu.org.uy/ publicaciones/libros/ejemplares_ii/art_25_burghi. pdf. [Consulta 19/12/2019].

(4) Soiza Larrosa A. Tte. Cnel. (M)(R) José Grunberg. Salud Mil 2012; 31(1):5-9.

(5) Uruguay. Ministerio de Defensa Nacional. Boletín $\mathrm{N}^{\circ} 6.229$. 